\title{
Quantum beat of strain-induced GaAs quantum dots
}

\author{
Kazuhiro Nishibayashi, Tsuyoshi Okuno, Ivan V. Ignatiev, \\ Serguei Verbin and Yasuaki Masumoto \\ Institute of Physics, University of Tsukuba, Tsukuba, Ibaraki \\ 305-8571, Japan
}

\begin{abstract}
We report the experimental observation of transient oscillation behaviour of the strain-induced GaAs quantum dots in the time-resolved photoluminescence in the magnetic field parallel to the growth direction. We regard the origin of the oscillation as quantum beats caused by quantum interference of excitons showing Zeeman splitting. We calculated the absolute g-factor of the quantum dots, and it was 0.47 .
\end{abstract}

\section{Introduction}

The semiconductor quantum dots (QD's), which are in the limit of the smallest size region, show the strong confinement. The quantized energy levels of QD's are compared with atomic-like states. Many investigations of optical transition in QD's have given us valuable information on fundamental physics, which is relevant to the development of new quantum devices. The quantum computation is one of the developing technologies. To realize this epoch-making technology that incorporates the conception of quantum dynamics in calculation, the study of fundamental physics of semiconductor, especially the spin dynamics, becomes important. Observation of quantum beats is known to be an efficient method for studying a fine energy structure and spin dynamics of carriers in quasi-2D structures. The observation of quantum beats under the magnetic field has been reported. Time-resolved absorption saturation measurement revealed the electron and hole g-factor in quantum well (QW) [1]. In the investigation of time-resolved photoluminescence measurement, quantum beats were observed under the resonant excitation of multiple QW's [2]. Time-resolved photoluminescence revealed hole spin quantum beats [3]. Although many observations of quantum beats have been reported, the observations of quantum beats for the QD's are limited. This is usually ascribed to the large inhomogeneous broadening in the ensembles of QD's.

The utilization of stress caused by lattice mismatch between different semiconductors is an ideal method for fabrication of QD's. In this method, QD's are formed in the QW by the stress modulation from self-assembled dots fabricated on the surface. Such QD's are called strain-induced quantum dots [4-7]. They have advantages of homogeneous size distribution in the growth direction and no defect on the interface. We report the first observation of quantum beats coming from the Zeeman splitting among the ensemble of strain-induced quantum dots. 


\section{Sample}

The samples were grown by metal organic vapor phase epitaxy at 60 Torr on semiinsulating GaAs (001) substrates. The GaAs/AlGaAs QW was grown on the substrate. The $\mathrm{Al}$ concentration of the barrier layer was $30 \%$. The GaAs QW layer is $3.9 \mathrm{~nm}$ thick. The AlGaAs top barrier layer was $9.6 \mathrm{~nm}$ thick. After the growth of the single QW, a 2.4 $\mathrm{nm}$ GaAs cap layer was grown. Finally, InP islands were formed by depositing 4 monolayer InP as stressors. The islands were $\sim 90 \mathrm{~nm}$ wide and $\sim 30 \mathrm{~nm}$ high. The areal density of the islands was approximately $3 \times 10^{9} \mathrm{~cm}^{-2}$.

\section{Experimental Details}

\subsection{PL and PLE measurement}

Figure 1(a) shows photoluminescence (PL) spectrum excited at $2.33 \mathrm{eV}$ by using a continuous-wave $\mathrm{Nd}: \mathrm{YVO}_{4}$ laser. The luminescence located at $1.642 \mathrm{eV}$ is attributed to electron-hole recombination in the GaAs QW without modulation by stress. In the lower energy region around $1.60 \mathrm{eV}$, the luminescence from strain-induced quantum dots states is observed. In the figure, QD1 and QD2 denote the first and the second excited states of dots, respectively. We measured photoluminescence excitation (PLE) spectrum to specify the energy states of the dots more precisely [8]. In these measurements, polarized PLE spectra are obtained. A Ti:sapphire laser was used for an exciting source, which is circularly polarized by a quarter wave plate. The luminescence components of the same $(\sigma(+))$ and the opposite $(\sigma(-))$ circular polarization as the excitation laser were selectively measured by rotating another quarter wave plate placed in front of a double monochromator. The luminescence intensity was measured by a charge-coupled-device camera with changing the wavelength of the exciting laser.

The circularly polarized PLE spectra are shown in Fig. 1(b). The detected energy position is set to the peak of QD1 $(1.597 \mathrm{eV})$. Several structures are observed. QD2 and QD3 denote the absorption by the second and the third excited states of the dots. There is a sharp peak at $1.632 \mathrm{eV}$. The energy difference between this peak and the detected energy position is $35 \mathrm{meV}$. That is equal to the LO-phonon energy of GaAs. Therefore, the origin of this peak is the luminescence through one LO-phonon relaxation. The peaks at $1.653 \mathrm{eV}$ and $1.680 \mathrm{eV}$ correspond to heavy-hole $(\mathrm{HH})$ exciton and light-hole (LH) exciton in QW, respectively. When we compare the results of $\sigma(+)$ and $\sigma(-)$ excitations, clear difference is detected. The PLE spectrum for $\sigma(+)$ is larger than that for $\sigma(-)$. The degree of circular polarization is as much as 0.4 at the LO phonon peak. When the $\mathrm{HH}$ state was excited, the difference of the intensity between $\sigma(+)$ and $\sigma(-)$ is enhanced. When the LH state was excited, the difference almost disappeared. It is concluded that the energy state of strain-induced quantum dots is originated from the $\mathrm{HH}$ state of QW and thus the polarization property of luminescence of QD is retained up to the HH state of QW. 


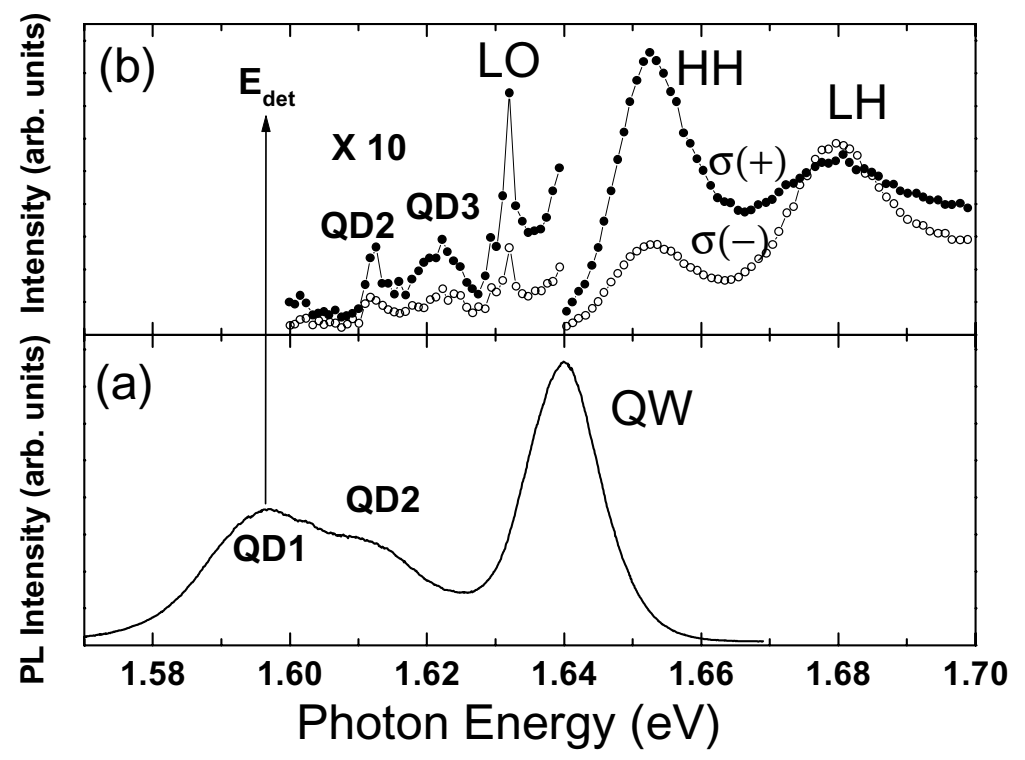

Figure 1. (a) Photoluminescence spectrum of GaAs quantum dots excited with a continuous wave $\mathrm{Nd}: \mathrm{YVO}_{4}$ laser. (b) Polarized photoluminescence excitation spectra. The detected energy position is set to the first excited state of the quantum dots, which is obtained from the PL measurement. The closed (open) circles show the same (opposite) circular polarization as the excitation laser

\subsection{Magnetophotoluminescence measurement}

The sample was mounted in a cryostat with a superconducting magnet at $5 \mathrm{~K}$. The direction of an external magnetic field is along the growth direction of epilayers. For the measurement of time-resolved photoluminescence, we used 2 4 ps pulses from a modelocked Ti:sapphire laser. The pulse repetition rate was $82 \mathrm{MHz}$. We used the backscattering geometry. The excitation and the detection paths were along the magnetic field. The excitation pulses were linearly polarized. The luminescence components with linear polarization parallel $(\|)$ or perpendicular $(\perp)$ to that of the excitation laser was selected by a half wave plate and a polarizer. The luminescence was dispersed by a subtractive-dispersion double monochromator and detected by a streak camera. The typical time resolution of the system is $\sim 10 \mathrm{ps}$.

The excitation energy was carefully selected based on the information obtained from the PLE measurements in Fig. 1. When the excitation energy above QW is used, the luminescence intensity of QD1 is high. However, most of QD's show luminescence. This condition might not be favourable for our time-resolved photoluminescence measurements, because luminescence of QD's involves substantial inhomogenity. When the excitation energy below QW is used, the luminescence intensity of QD1 becomes very weak rapidly. This is because the light absorption by a single layer of QD's is much weaker than the light absorption by the thick barrier layers. However, resonant excitation of QD's by narrow-band picosecond pulses site-selectively excites an ensemble of QD's of almost the same size. Further, the photoluminescence intensity of QD1 is enhanced by 
the LO phonon resonance. From these reasons, we used the sum of the QD1 energy and the LO phonon energy for the excitation energy (see the inset in Fig. 2(a)).

Figure 2(a) shows the time-resolved photoluminescence at $\mathrm{B}=0 \mathrm{~T}$. The solid curve was measured at parallel polarization. The decay time is obtained to be around $30 \mathrm{ps}$. After the photoexcited electron-hole pairs relax to QD1 with emitting LO phonon immediately, they recombine and show luminescence. The intensity of parallel component corresponds to the number of carriers that conserve the polarization memory in the LO phonon relaxation. The dotted curve was measured at perpendicular polarization. The difference of these curves shows that the degree of polarization is $\sim 0.2$ around the luminescence peak.
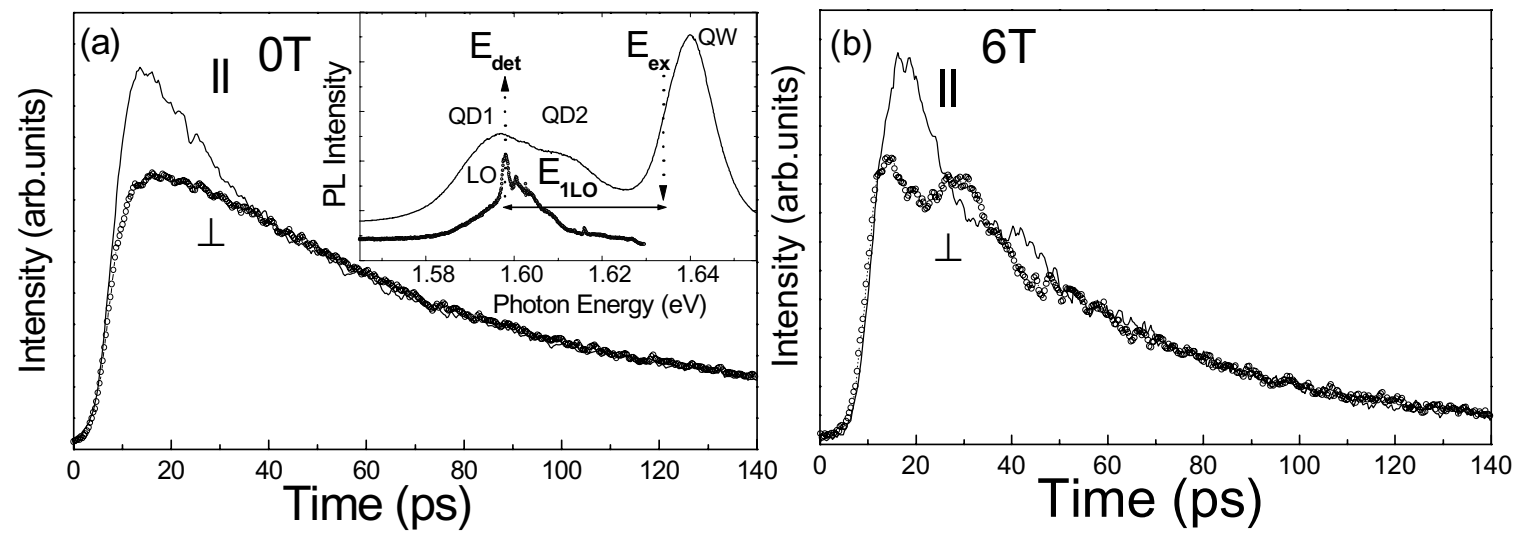

Figure 2. Time-resolved photoluminescence profiles with co- and crosslinear polarization at $0 \mathrm{~T}(\mathrm{a})$ and $6 \mathrm{~T}$ (b). The solid (dotted) curve show the co-(cross-)linear polarization component of luminescence. The inset in (a) shows selectively excited photoluminescence spectrum (thick line) and non-selectively excited photoluminescence spectrum. $\mathrm{E}_{\mathrm{ex}}$ and $\mathrm{E}_{\mathrm{det}}$ show the excitation and detection energy for time-resolved photoluminescence measurements. The difference of these energies corresponds to the LO phonon.

When a magnetic field is applied, a damping oscillation structure appeared upon the decay profile. Figure $2(\mathrm{~b})$ shows the result measured at $\mathrm{B}=6 \mathrm{~T}$. The solid curve denotes the parallel polarization and the dotted curve denotes perpendicular polarization. The parallel component decreases faster than that at $\mathrm{B}=0 \mathrm{~T}$, and is followed by undulating oscillations. Still more, dramatic changes were observed on the perpendicular component. After the quick decrease around $t \sim 12$ ps in the horizontal axis, the remarkable pulsation appeared. The parallel and perpendicular polarizations are antiphase and the oscillations of these two were levelled off after 60 ps.

Further, we measured the time-resolved photoluminescence at several magnetic field strengths and investigated degree of polarization 
$\mathrm{P}=(\mathrm{I}(\|)-\mathrm{I}(\perp)) /(\mathrm{I}(\|)+\mathrm{I}(\perp))$.

The result is displayed in Fig. 3. The magnetic field was changed from 0 to $8 \mathrm{~T}$. As the applied magnetic field strength increases, the oscillation frequency is shortened. The number of oscillations that can be resolved increases in number from two at $2 \mathrm{~T}$ to four at $8 \mathrm{~T}$.

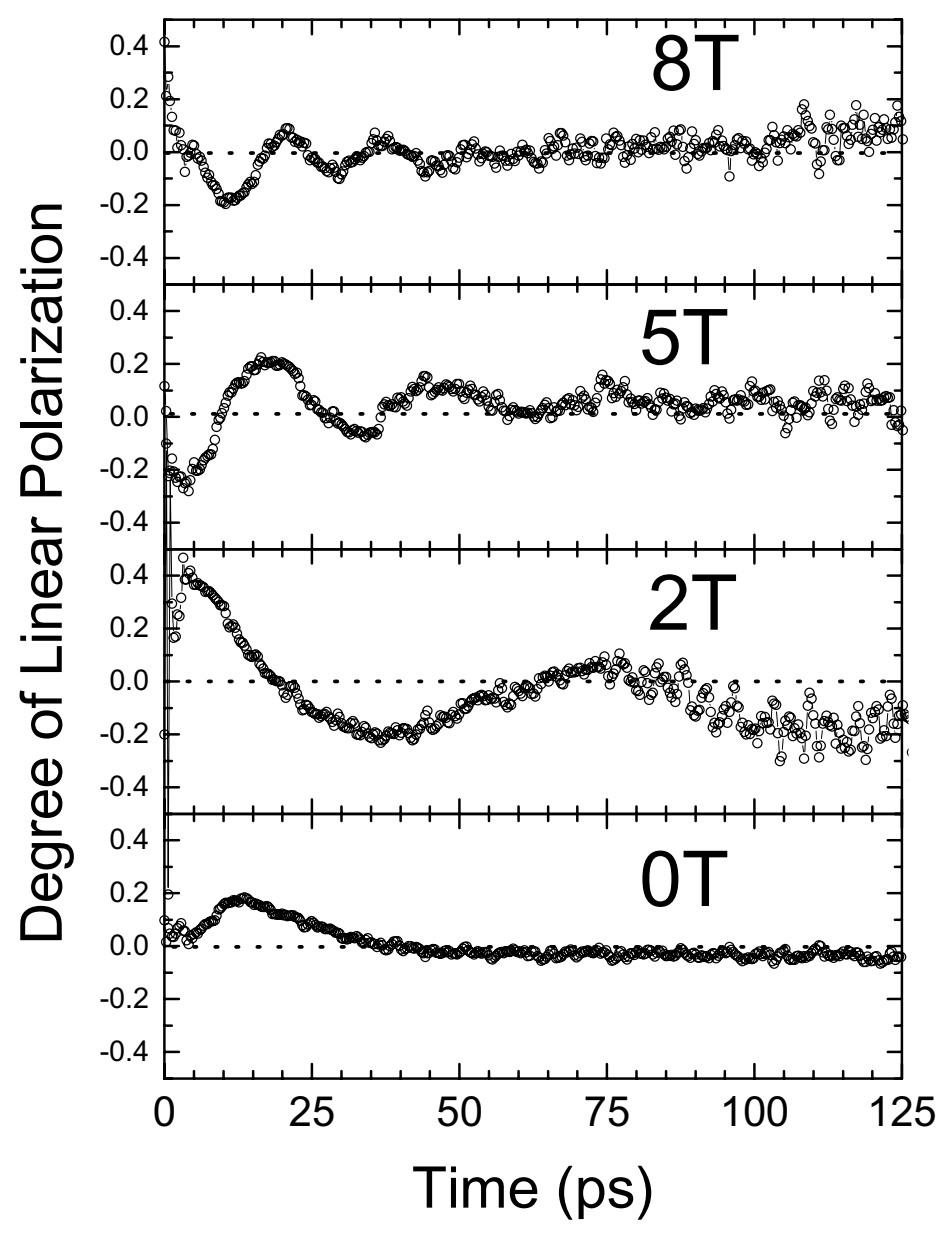

Figure 3. Degree of linear polarization at different magnetic field strength.

For the analysis, we fitted these degrees of polarization to the formula

$$
I=\exp (-t / \tau) \sin (\omega t)
$$

Where, $\omega$ means the angular frequency of pulsation, and $\tau$ means the decay time. 
An instance is shown in the inset of Fig. 4. The magnetic field strength is $6 \mathrm{~T}$. The solid curve is the fitted function. From this calculation, the period is estimated to be $25 \mathrm{ps}$. The period of the beats decreased with increasing magnetic field.

We considered that the beat is attributed to the Zeeman splitting. To verify this assumption, we calculated the energy separation from the periods at various magnetic fields. Figure 4 shows the result. The calculated value was proportional to the magnetic field. The energy separation at $8 \mathrm{~T}$ was $0.217 \mathrm{meV}$. We made an interpretation for the origin of the oscillation in time-resolved photoluminescence. The obtained splitting is much smaller than the laser linewidth $(0.7 \mathrm{meV})$. The interference among the fluorescence from the ensemble of QD's is small. From this point of view, we regard the periodical oscillation in the time-resolved photoluminescence as the quantum interference caused by the Zeeman splitting in a quantum dot. We calculated the absolute value of the g-factor of the QD's, and it was 0.47.

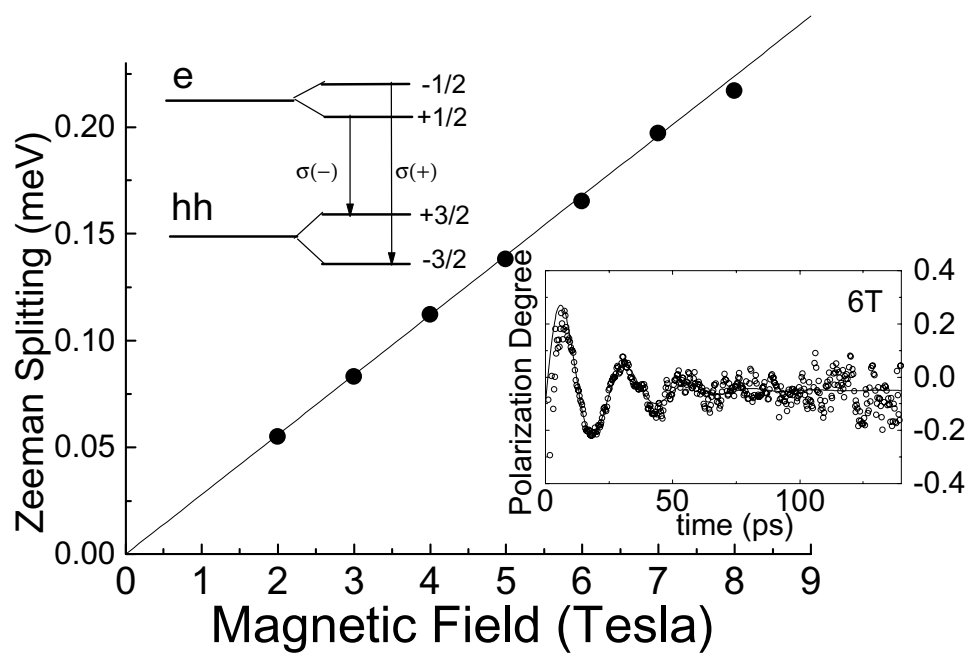

Figure 4. The observed Zeeman splitting (closed circles). The g-factor is 0.47 . The inset shows degree of polarization obtained at $6 \mathrm{~T}$ (open circles). The solid curve is a fit. A scheme shows optical transition from Zeeman components of QD1 state. 


\section{References}

[1] Bar-Ad S and Bar-Joseph I 1991 Phys. Rev. Lett 66 2491-2494

[2] Amand T, Marie X, Jeune P Le, Brousseau M, Robert D, Barrau J and Planel R 1997 Phys. Rev. Lett 78 1355-1358

[3] Marie X, Amand T, Jeune P Le, Paillard M, Renucci P, Golub L E, Dymnikov V D and Ivchenko L E 1999 Phys. Rev. B 60 5811-5817

[4] Lipsanen H, Sopanen M and Ahopelt 1995 Phys. Rev. B 51 13868-13871

[5] Tulkki J and Heinämäki A 1995 Phys. Rev. B 52 8239-8243

[6] Grosse S, Sandmann J H H, Plessen G von, Feldmann J, Lipsanen H, Sopanen M, Tulkki J and Ahopelto J 1997 Phys. Rev. B 55 4473-4476

[7] Rinaldi R, Giugno P V, Cingolani R, Lipsanen H, Sopanen M, Tulkki J, and . Ahopelto J 1996 Phys. Rev. Lett. 342-345

[8] Nishibayashi K, Okuno T, Mishina T, Sugou S, Ren H-W and Masumoto Y 2001 Jpn. J. Appl. Phys. 40 2084-2086 